\title{
MATRIX SUMMABILITY OF UNBOUNDED SEQUENCES
}

\author{
J. DEFRANZA AND K. ZELLER
}

(Communicated by Andrew M. Bruckner)

\begin{abstract}
A well-known result of Mazur and Orlicz states that a matrix method strictly stronger than convergence sums not only bounded sequences but unbounded sequences. We consider the question of whether a matrix method strictly stronger than convergence will also sum a sequence with series terms (differences) constituting an unbounded sequence. This is equivalent to the series to sequence convergence domain of the matrix containing an unbounded sequence. A simple criterion is given showing in many cases the answer is positive. Counterexamples of three types are considered; triangles that are not perfect, perfect row finite matrices, and perfect triangles.
\end{abstract}

\section{INTRODUCTION}

It is well-known that a matrix method that is strictly stronger than convergence sums not only bounded sequences but unbounded sequences. We consider the question of whether a matrix method strictly stronger than convergence will also sum a sequence whose series terms (differences) constitute an unbounded sequence. We refer to such sequences briefly as series with unbounded terms. The answer is positive in many cases as is shown by Lemma 3.3. Counterexamples of three types are given in Propositions 4.1 and 4.2 and in Theorem 5.1 respectively. Propositions 4.1 and 4.2 deal with triangles that are not perfect (Einfolgenverfahren) and with perfect row-finite methods (generated by intertwining the rows of two matrices) respectively. A more delicate construction yields a suitable triangle that is also perfect (Theorem 5.1). In $\S 6$ we conclude with several remarks.

\section{NOTATION AND TERMINOLOGY}

Throughout we use notation and results given in the texts by Wilansky [11] and by Zeller-Beekmann [15]. Let $\omega$ denote the space of all sequences, $m$ all bounded sequences, $c$ the convergent sequences, $c_{0}$ sequences that converge to $0, c s=\left\{x: \sum_{n} x_{n}\right.$ is convergent $\}, l_{1}=\left\{x: \sum_{n}\left|x_{n}\right|<\infty\right\}$, and $\varphi$ all finitely nonzero sequences. If $A=\left(a_{n k}\right)$ is an infinite matrix, the matrix method $A$ defines a sequence to sequence transformation, mapping the sequence $s$ (real

Received by the editors November 19, 1990 .

1991 Mathematics Subject Classification. Primary 40C05, 40G99, 40H05, 46A45. 
or complex terms) to $t$

$$
t_{n}=(A s)_{n}=\sum_{k=0}^{\infty} a_{n k} s_{k}, \quad n=0,1,2, \ldots
$$

The convergence domain $c_{A}$ for the matrix method $A$ consists of those sequences $s$ for which $t=A s$ exists and belongs to $c$. The $A$-limit is defined for $s \in c_{A}$ by $A-\lim s_{k}=\lim t_{n}$. The method $A$ is called conservative provided $c \subset c_{A}$ and strictly stronger than convergence if, moreover, $c \neq c_{A}$. A conservative matrix $A$ is regular provided $A$ - $\lim s_{k}=\lim s_{k}$ for all $s \in c$ and is perfect if $c$ is dense in the FK space $c_{A}$, which is equivalent to the sequences $e=(1,1,1, \ldots), e^{0}=(1,0,0, \ldots), e^{1}=(0,1,0, \ldots)$, etc. forming a fundamental set, see for example [15]. The matrix $A$ is a triangle provided $a_{n k}=0$ for $k>n$ and $a_{n n} \neq 0$ for all $n$. Let $\Sigma$ denote the triangle of all ones, so that $A \Sigma$ is the series to sequence matrix method associated with $A$. Together with each $s$ we consider the corresponding $u$ given by the series terms (differences), i.e., $s_{k}=\sum_{i=0}^{k} u_{i}$ for all $k$. Thus we have $s=\sum u$ and $u=\sum^{-1} s$. We say that the method $A$ has SB if each $s \in c_{A}$ is bounded (i.e., $c_{A} \subseteq m$ ) and has UB, respectively UN, if for each $s \in c_{A}, u=\sum^{-1} s$ is bounded (i.e, $c_{A \Sigma} \subseteq m$ ), respectively $u \in c_{0}$ (i.e., $c_{A \Sigma} \subseteq c_{0}$ ). We remark here that UB and perfectness imply UN (by the fundamental set principle, see [15, Result 16 I]).

\section{CRiteria}

The following known result is our starting point.

Lemma 3.1. A matrix method $A$ that is strictly stronger than convergence violates $S B$ (i.e., $c_{A}$ contains unbounded sequences).

This result goes back to Mazur and Orlicz, see [15, Result 29 I].

The next result exhibits a relation between the properties SB and UB. The row-norms of the matrix method $A$ are given by $\sum_{k}\left|a_{n k}\right|$ for each $n \geq 0$.

Lemma 3.2. A triangle $A$ has $S B$, respectively $U B$, if and only if the row-norms of $A^{-1}$, respectively $\Sigma^{-1} A^{-1}$, are bounded.

The proof of the lemma is standard going back to Toeplitz. See for example [15, Result 32 I] or [11, Theorem 3.3, p. 5, Theorem 5, p. 12].

The final lemma of this section is applicable to many common methods.

Lemma 3.3. If $\underline{\lim }_{k} \sup _{n}\left|a_{n k}\right|=0$ then the matrix method $A$ violates $S B$ and $U B$. If $A$ is a triangle and $\underline{\lim }_{k}\left|a_{k k}\right|=0$ then $A$ violates $S B$ and $U B$.

The first part follows from [15, Result 25 I] (Spaltenmaximumktiterium), this criterion going back to Agnew [1]. For the second part apply Lemma 3.2, see [11, Theorem 7, p. 12].

\section{EXAMPLES}

The next two propositions exhibit two classes of matrix methods with property UB. 
Proposition 4.1. There is a regular matrix method A strictly stronger than convergence having the following properties: The matrix is a triangle, the method is not perfect, and the method satisfies $U B$.

Proof. Given any unbounded sequence $d$, there is a regular triangle $A$ such that $c_{A}$ consists of sequences of the form $\alpha d+y$ where $y \in c, \alpha \in \mathbb{C}\left(c_{A}=c \oplus\right.$ $d$ ), see [15, Result $26 \mathrm{II}]$ (Allgemeine Einfolgenverfahren). The result goes back to Mazur [8], Darevsky [3], and Zeller [14]. Here we choose any unbounded sequence $d$ with differences bounded. Then $c_{A \Sigma}=c s \oplus \Sigma^{-1} d \subseteq m$.

Proposition 4.2. There is a regular matrix method A strictly stronger than convergence having the following properties: The matrix is row-finite, the method is perfect, and the method satisfies UB.

Proof. We begin with a row-finite matrix $B$ and $C=B \Sigma$. Thus $t=B s=C u$ (sequence and series forms respectively). Intertwining the rows of $C$ and $C+\mathrm{I}$ (I the identity matrix) we obtain a matrix $D$ and $A=D \Sigma^{-1}$ such that $c_{A}$ consists of the $s \in c_{B}$ with $u \in c_{0}$. That is, the series domain $c_{D}$ equals $c_{C} \cap c_{0}$. As is well known $c_{C}$ and $c_{0}$ are BK-spaces with norms $\sup _{n}\left|t_{n}\right|$ and $\sup _{m}\left|u_{m}\right|$ respectively. The space $c_{D}$ is then a BK-space with the sum of these two norms. Now let $B$ be the Cesàro matrix of order one. Then $A$ is regular and strictly stronger than convergence (there are divergent Cesàro summable series that are in $c_{0}$ ). Finally we show that $A$ is perfect. The BK-space $c_{C}$ has $C$-sectional convergence. i.e.,

$$
\lim _{n}\left\|c_{n 0} u_{0} e^{0}+c_{n 1} u_{1} e^{1}+\cdots+c_{n n} u_{n} e^{n}-u\right\|_{c_{C}}=0
$$

for each Cesàro summable series $u$ (by Hardy-Bohr, see [14, 7]). Since the BK-space $c_{0}$ has AK (Abschnittskonvergenz), i.e., $\left\{e^{n}\right\}_{n \geq 0}$ is a basis, it follows that $c_{0}$ also has $C$-sectional convergence. Together this yields $C$-sectional convergence in the BK-space $c_{D}$. In particular, $c_{D}$ will then have $\mathrm{AD}(\mathrm{Ab}-$ schnittsdiche), i.e., $\varphi$ is dense, and it follows that the matrix $A$ is perfect.

\section{MAIN RESUlt}

We now combine the properties "triangle" and "perfect" that appear separately in the examples of $\S 4$. The construction here is more delicate and allows for many modifications.

Theorem 5.1. There is a regular matrix method $A$ strictly stronger than convergence having the following properties: The matrix is a triangle, the method is perfect, and the method satisfies $U N$.

Proof. We use the matrix $Z=Z_{1 / 2}$ (Zweierverfahren), see [15, p. 125], spread it and insert suitable rows. Our precise definition of the triangle $A$ begins with $a_{00}=a_{10}=a_{11}=\frac{1}{2}$. Then we consider a fixed $j \geq 1$ and define $p=(j-1)^{2}$, $q=j^{2}, r=(j+1)^{2}, \delta=1 / 4 j$, and

$$
t_{n}=\alpha_{n} s_{p}+\beta_{n} s_{q}+\frac{1}{2} s_{n} \quad \text { for } q<n \leq r,
$$

where

$$
\alpha_{n}+\beta_{n}=\frac{1}{2}, \quad \alpha_{q+1}=\frac{1}{2}, \quad \alpha_{q+2}=\frac{1}{2}-\delta, \quad \alpha_{q+3}=\frac{1}{2}-2 \delta, \ldots, \alpha_{r}=0 .
$$


For $n=r$ we have a row as in $Z$, with the terms spread. We consider

$$
t_{n}-t_{n-1}=\left(\alpha_{n}-\alpha_{n-1}\right) s_{p}+\left(\beta_{n}-\beta_{n-1}\right) s_{q}+\frac{1}{2}\left(s_{n}-s_{n-1}\right)
$$

$\left(u_{n}=s_{n}-s_{n-1}\right)$ where in this relation we let $\alpha_{p}=\frac{1}{2}$ and $\beta_{q}=0$. For $s \in c_{A}$ the first two terms on the right-hand side are $o(1)$ by the definition of the coefficients and since $s_{j^{2}}=o(j)$ (use the order condition for $Z$ ). Thus $u \in c_{0}$. Further $A$ is strictly stronger than convergence (take e.g., $s_{j^{2}}=(-1)^{j}$ and define the remaining $s_{k}$ by $t_{k}=0$ ).

Finally to show that $A$ is perfect we use [15, Result $23 \mathrm{I}]$ (or see [11, Theorem 4, p. 42]). That is, we show that $A$ is of type $M$. Thus suppose $g \in l_{1}$ and $g A=0$. It is immediate that $g_{n}=0$ for $n \neq j^{2}, j \geq 0$. Moreover, it follows that for $n=j^{2}, j \geq 0, g_{n}=(-1)^{j} g_{0}$, and hence the terms must vanish for any $g \in l_{1}$. Thus we see that $A$ is perfect.

\section{REMARKS}

The Nörlund polynomial methods provide a collection of matrix methods that, when strictly stronger than convergence, are easily seen to violate UB.

For a polynomial $p(z)=\sum_{n=0}^{N} p_{n} z^{n}$ of degree $N$ the Nörlund polynomial method $N_{p}$ is given by $N_{p}[n, k]=p_{n-k}$ for $k \leq n$ and 0 otherwise where $p_{n}=0$ for $n>N$. If $p(z)=\prod_{i=1}^{N}\left(a_{i}+b_{i} z\right)$, since Nörlund polynomial methods commute, then for each $i, 1 \leq i \leq N, c_{N_{q_{i}}} \subseteq c_{N_{p}}$ where $q_{i}(z)=$ $a_{i}+b_{i} z$. Without loss of generality we may consider only polynomial methods generated by polynomials of the form $p(z)=a+b z$. We may also assume $|a / b| \leq 1$, for otherwise the method will be equivalent to convergence, see [5, Theorem 4.6]. Moreover, $N_{p}$ is of type $M$, and hence perfect, see [11, Theorem 4, p. 42], if and only if $|a / b| \geq 1$, see [4]. If $N_{p}$ is generated by $p(z)=a+b z$ and is strictly stronger than convergence by Lemma 3.2, $N_{p}$ violates UB and consequently sums a series with unbounded terms, see also [6]. Hence by the previous remarks Nörlund polynomial methods strictly stronger than convergence violate UB. This can be extended to include a class of little Nörlund means. That is, let $p(z)=\sum_{n=0}^{\infty} p_{n} z^{n}$; then the little Nörlund mean $N_{p}$ associated with this series is given by $N_{p}[n, k]=p_{n-k}$ for $k \leq n$ and 0 otherwise. If the radius of convergence of the power series is greater than one and $p(\alpha)=0$, then $p(z)=(z-\alpha) q(z)$ where $q(z)=\sum_{k=0}^{\infty} q_{k} z^{k}$, with the radius of convergence of $q(z)$ also greater than one. Moreover, $q \in l_{1}$ and $N_{p}=N_{q} N_{r}$ where $r(z)=(z-\alpha)$. If $N_{p}$ is stronger than convergence then there must exist some $\alpha$ with $|\alpha| \leq 1$, and the result now follows.

\section{REFERENCES}

1. R. P. Agnew, A simple sufficient condition that a method of summability be stronger than convergence, Bull. Amer. Math. Soc., 52 (1946),128-132.

2. __ Equivalence of methods for evaluation of sequences, Proc. Amer. Math. Soc. 3 (1952), 550-556.

3. V. Darevsky, On intrinsically perfect methods of summation, Bull. Acad. Sci. URSS Ser. Math. (Izv. Akad. Nauk SSSR Ser. Mat.) 10 (1946), 97-104.

4. J. DeFranza and D. J. Fleming, Nörlund polynomial methods of type $M(E)$, Houston J. Math., 10 (1984), 371-381. 
5. __ Nörlund Polynomial Methods on BK Spaces, Houston J. of Math. 9 (1983), 447-453.

6. __ On the Bohr Hardy criteria, Arch. Math. 51 (1988), 464-473.

7. G. G. Lorentz and K. Zeller, Abschnittslimitierbarkeit und der Satz von Hardy-Bohr, Arch. Math. 15 (1964), 213-288.

8. S. Mazur, Über lineare limitierungsverfahren, Math. Z. 28 (1928), 599-611.

9. S. Mazur and W. Orlicz, Sur les méthodes linéaires de sommation, C. R. Acad. Sci. Paris Sér. I Math. 196 (1933), 32-34.

10. A. K. Snyder and A. Wilansky, Inclusion theorems and semi-conservative FK spaces, Rocky Mountain J. Math. 2 (1972), 595-603.

11. A. Wilansky, Summability through functional analysis, North Holland, Amsterdam, 1984.

12. A. Wilansky and K. Zeller, Summation of bounded divergent sequences, Trans. Amer. Math. Soc. 78 (1955), 501-509.

13. K. Zeller, Approẍimation in Wirkfeldern von Summierungsverfahren, Arch. Math. 4 (1953), 425-431.

14. __ Merkwürdigkeiten bei Matrixverfahren; Einfolgenverfahren, Arch. Math. 4 (1953), $1-5$.

15. K. Zeller and W. Beekmann, Theorie der Limitierungsverfahren, Springer-Verlag, Berlin, Heidelberg, and New York, 1970.

Mathematics Department, St. Lawrence University, Canton, New York, 13617

Mathematische Fakutät, Universität Tübingen, Auf der Morgenstelle 10, D-7400, TÜBINGEN, WeSt GeRMANY 